\title{
A single vector-based strategy for marker-less gene replacement in Synechocystis sp. PCC 6803
}

\author{
Stefania Viola, Thilo Rühle and Dario Leister ${ }^{*}$
}

\begin{abstract}
Background: The cyanobacterium Synechocystis sp. PCC 6803 is widely used for research on photosynthesis and circadian rhythms, and also finds application in sustainable biotechnologies. Synechocystis is naturally transformable and undergoes homologous recombination, which enables the development of a variety of tools for genetic and genomic manipulations. To generate multiple gene deletions and/or replacements, marker-less manipulation methods based on counter-selection are generally employed. Currently available methods require two transformation steps with different DNA plasmids.

Results: In this study, we present a marker-less gene deletion and replacement strategy in Synechocystis sp. PCC 6803 which needs only a single transformation step. The method utilizes an nptl-sacB double selection cassette and exploits the ability of the cyanobacterium to undergo two successive genomic recombination events via double and single crossing-over upon application of appropriate selective procedures.
\end{abstract}

Conclusions: By reducing the number of cloning steps, this strategy will facilitate gene manipulation, gain-of-function studies, and automated screening of mutants.

Keywords: Genetic engineering, Gene replacement, Homologous recombination, Synechocystis

\section{Background}

Cyanobacteria, also known as blue-green algae, are Gram-negative, photosynthetic prokaryotes. Moreover, they are the evolutionary ancestors of plastids [1] and the only prokaryotes that exhibit a classical circadian clock mechanism [2]. Thanks to their ability to adapt to extreme environmental conditions, cyanobacteria are widely distributed. These features, and their recent exploitation for biotechnological purposes [3,4], explain why cyanobacteria have been extensively studied. Over the past few decades, numerous cyanobacterial genomes have been sequenced (http://genome.microbedb.jp/cyanobase) and many molecular tools for their genetic manipulation have been developed. Synechocystis sp. PCC 6803 (hereafter designated as Synechocystis) is an excellent model organism, because it has a small $(3.6 \mathrm{Mb})$, sequenced genome [5], is capable of spontaneous uptake of exogenous DNA and can integrate it via homologous recombination into its genome. In addition, a spontaneous glucose-tolerant mutant is available [6] that can

\footnotetext{
*Correspondence: leister@lmu.de

Department Biology I, Ludwig-Maximilians-Universität München,

Großhaderner Str. 2, Planegg, Martinsried D-82152, Germany
}

grow heterotrophically in the presence of glucose and is thus useful for the study of oxygenic photosynthesis. Photosynthetic Synechocystis mutants have been extensively used to study photosystems I (PSI) $[7,8]$ and II (PSII) $[9,10]$ and to investigate the functionality of photosynthesis-related proteins from higher plants in cyanobacteria [11,12]. All these studies have employed gene deletions or replacements.

The classical means of deleting a target gene or inserting exogenous genetic material into the Synechocystis genome via homologous recombination involves the use of a resistance marker $[9,13]$. In this approach, the insertion cassette (marker plus exogenous DNA) is flanked by sequences homologous to the targeted site in the genome, and the whole segment is cloned into a suicide vector, which is unable to replicate in the host cell. After integration of the insertion cassette into the host genome, the resistance-mediating marker allows for positive selection of the mutant cells in which integration has occurred. However, to perform additional genomic modifications, one must use other resistance markers. Consequently, the number of available markers restricts the number of genetic manipulations. To overcome this 
limitation, so called marker-less strategies have been developed, which allow for the removal of the integrated marker. The first marker-less method was established in Gram-negative bacteria using the nptI-sacB double selection cassette [14]. Here, the nptI gene confers resistance to the antibiotic kanamycin, while expression of the $s a c B$ gene from Bacillus subtilis $[15,16]$ is toxic to Gram-negative bacteria grown on sucrose-containing media. SacB is a levansucrase that hydrolyzes sucrose, and utilizes the fructose unit for the production of levans [17]. The polymers are toxic to Gram-negative bacteria, and cells harbouring the $s a c B$ gene die when grown in presence of $5 \%$ sucrose, although the underlying mechanism is not fully understood. In cyanobacteria, the $n p t I-s a c B$ cartridge was first used to establish marker-less gene replacement in Anabaena sp. PCC 7120 [18]. Gene replacement requires two homologous recombination events, each of which requires a suicide vector and a bacterial transformation step (see Additional file 1). 1) The target genomic sequence is replaced by the double-selection cassette, and the mutants in which the homologous recombination has taken place are positively selected by their ability to grow in the presence of the antibiotic. 2) A second transformation and homologous recombination event leads to the excision of the nptI-sacB selection cartridge and its concomitant replacement by the exogenous sequence. The desired mutants can be selected on the basis of their resistance to sucrose and sensitivity to kanamycin. An alternative version of double selection was developed for gene replacement in Synechococcus elongatus PCC 7942 [19]. In this approach, the background strain carries a mutated form of the rps12 gene (which codes for subunit S12 of the 30S ribosome) which confers resistance to spectinomycin [20,21]. Since the mutation is recessive, the double selection cassette carries a kanamycin resistance gene and - as an alternative negative selection marker - a wild-type copy of rps12, which results in a dominant spectinomycin-sensitive phenotype. A drawback of the rps12 marker system is the need to perform all multiple replacements in the rps12 mutant background, which introduces the need of an additional manipulation step. A general weakness of the marker-less gene replacement methods described above is that two time-consuming transformation events and cloning of two different suicide vectors are required. Alternative double recombination strategies have been developed that rely on initial integration of the whole vector into the target genomic sequence via single crossing-over, as in the case of allelic exchange $[22,23]$. However, in this case, the second recombination, leading to the excision of the integrated vector, gives rise to both (reverted) wild-type cells and bacteria that retain the desired mutation, so that this population of recombinants must be screened by PCR.
The aim of the present study was to develop a timesaving strategy for marker-less gene replacement in Synechocystis which requires only a single vector and a single bacterial transformation event. The strategy finally chosen leads to a population which consists exclusively of mutants. Therefore, the method offers a number of technical advantages over the classical approaches and is applicable to other bacterial species capable of undergoing gene transfer, irrespective of the selective markers employed.

\section{Results}

\section{Design of the single-step double-recombination vectors}

A vector designed for a single-step double-recombination approach (see Figure 1) must include several essential elements: 1. an origin of replication for E. coli that is nonfunctional in Synechocystis; 2. flanking sequences homologous to Synechocystis chromosomal sites to allow stable integration of the constructs into the cyanobacterial genome (designated as HR1 and HR2); 3. the nptI-sacB double selection cassette that allows both positive and negative selection of the Synechocystis recombinants; 4 . the exogenous gene of interest (GOI), split into two, partially overlapping (shaded box) segments (5' GOI and 3' GOI) that are separated by the $n p t I-s a c B$ cassette; 5 . a Synechocystis promoter for expression of the introduced recombinant gene(s).

When Synechocystis cells are transformed with such a vector, the initial recombination event takes place (Figure 1, upper panel) via a double crossover event between the two flanks of the construct, HR1 and HR2, and the corresponding homologous sequences present in the cyanobacterial genome. Mutants that have integrated the construct in this way contain the $n p t I-s a c B$ cassette in the target locus and can be positively selected on kanamycin. These recombinants are resistant to the antibiotic but sensitive to sucrose. In these mutants the GOI is not functional, because its sequence is disrupted by the $n p t I-s a c B$ cassette. Moreover, complete segregation of the first-round ["prim(ary)"] recombinants leads to loss of the endogenous target gene. The loss of the target gene also proves that integration of the construct happened via a double crossover event on HR1 and HR2 and not via a single crossover event involving only one flanking sequence. After complete segregation, the positive selective pressure is released and the mutants are grown in the absence of selection in order to allow the second recombination event to occur (Figure 1, lower panel), without the need for a further transformation step. The second crossover event occurs between the internal overlapping sequences of the foreign DNA and leads to the excision of the double selection cassette, restoring the integrity of the introduced gene(s). Mutants in which the second recombination is incomplete can be 


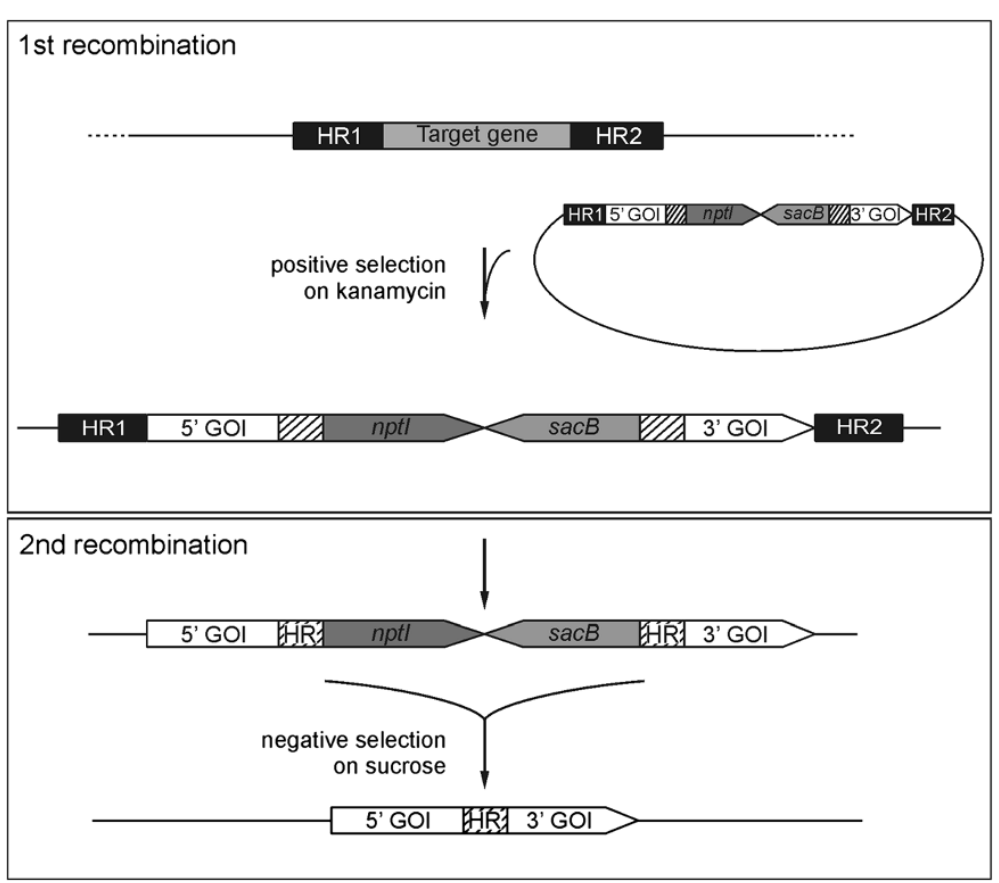

Figure 1 Schematic depiction of the single-step double recombination strategy. The first recombination step (upper panel), involving a double crossover between the homologous regions HR1 and HR2 of the vector and the genomic target sequence, leads to genomic integration of the construct. Note that the integrated gene of interest (GOI) is split into two parts, $5^{\prime}$ and $3^{\prime}$, the sequences of which partially overlap (shaded box). The $5^{\prime}$ and $3^{\prime} \mathrm{GOI}$ segments are separated from each other by the nptl-sacB selection cassette, which renders the first recombinant mutants resistant to kanamycin and sensitive to sucrose. After complete segregation of the replacement under positive selection in the presence of kanamycin, which ensures the total elimination of the endogenous target gene function, release of the selective pressure allows the second recombination to take place (lower panel). In this step, a crossover involving the overlapping regions of the split GOl leads to the excision of the $n p t /-s a c B$ cassette. Negative selection on sucrose yields colonies that have lost the entire sacB marker and carry the intact, functional GOI in place of the endogenous target gene.

efficiently counter-selected on sucrose, because only those recombinants that have lost the whole $n p t I-s a c B$ cassette will survive. In consequence, in the population generated by the second round of recombination ["sec (ondary) recombinants"], the target gene is replaced by the transgenic DNA of choice and no selection marker remains in the genome.

\section{Confirmation of the strategy: 1 . Introduction of the luciferase reporter system}

WT Synechocystis cells were transformed with the vector pDSlux (Figure 2A). The mutants obtained after the first round of recombination, lux ${ }^{\text {prim }}$, harboured an interrupted $\operatorname{lu} x A B$ operon inserted in the Synechocystis slr0168 ORF. After complete segregation, all the analyzed strains were knockouts for the slr0168 target locus, thus confirming the correct double crossover event in the first recombination step. In the mutants obtained after the second recombination round, named $l u x^{s e c}$, excision of the double selection cassette led to the reconstitution of the intact luxAB operon under the control of $\mathrm{P}_{p s b A 2}$.
The frequencies of the first and second recombination events were calculated for $l u x$ mutants. Ten independent lux $x^{\text {prim }}$ strains obtained after the first recombination were selected and allowed to undergo a second recombination. The transformation frequency was $4 \times 10^{-5}$, which is comparable to the values reported in the literature [24]. The frequency of the second recombination varied between $2 \times 10^{-5}$ and $1 \times 10^{-7}$ (Table 1), with an average value of $9 \times 10^{-6}$, revealing that it occurs with a frequency about ten times lower than the first. All the analyzed second-round recombinants were not able to grow in the presence of kanamycin, confirming that the sucrose resistance was due to loss of the double selection cassette and not to inactivation of the $s a c B$ gene by spontaneous mutations. Five of the original ten independent $l u x^{\text {prim }}$ first recombinants and two $l u x^{\text {sec }}$ second recombinants for each of the five $l u x^{\text {prim }}$ clones were selected for further analyses.

To confirm the correct segregation of the expected genetic variants in these mutants, genomic PCR was performed using three primer pairs. In all five lux prim mutants, but in none of the $l u x^{s e c}$ strains, the primer pairs $\mathrm{P} 1+\mathrm{P} 2$ and $\mathrm{P} 3+\mathrm{P} 4$ generated amplicons (2.7 and 





Table 1 Frequency of the second recombination event in independent lux ${ }^{\text {prim }}$ strains

\begin{tabular}{|c|c|c|c|c|c|c|c|c|c|c|}
\hline $\operatorname{lu} x^{\mathrm{sec}}$ & $\# 1$ & \#2 & \#3 & $\# 4$ & \#5 & \#6 & $\# 7$ & \#8 & $\# 9$ & $\# 10$ \\
\hline & $7 \times 10^{-6}$ & $5 \times 10^{-7}$ & $7 \times 10^{-6}$ & $4 \times 10^{-6}$ & $7 \times 10^{-6}$ & $1 \times 10^{-7}$ & $2 \times 10^{-6}$ & $2 \times 10^{-5}$ & $2 \times 10^{-5}$ & $2 \times 10^{-5}$ \\
\hline
\end{tabular}

2.3 kbps, respectively) spanning the 5' or 3' regions respectively of the $n p t I-s a c B$ cassette and the flanking segment of the interrupted $\operatorname{lux} A B$ operon. When combined, primers $\mathrm{P} 1$ and $\mathrm{P} 3$ amplified the reconstituted $\operatorname{lu} x A B$ operon, generating a PCR product of $2.3 \mathrm{kbps}$ that was detectable only in the $l u x^{s e c}$ second recombinants. The slr0168 downstream region (HR2, $\sim 1 \mathrm{kbps}$ ), used as a positive control, could be amplified in all the samples using the primer pair P5 + P6 (Figure 2B).

Gene replacement via homologous recombination is sometimes prone to error, and may result in nonhomologous DNA integration into the genome, thus generating mutants with multiple or non-targeted genomic insertions. However, when Southern blots bearing genomic XmaI digests from WT and the selected lux mutants were probed with a fragment of the $\operatorname{lu} x A B$ recombinant operon (Figure 2C), two labelled bands (F1 and F2, with 7.5 and $8.3 \mathrm{kbps}$ respectively) corresponding to the split $\operatorname{lux} A B$ operon were found in the lux $x^{\text {prim }}$ mutants, while the detection of a single band of $11 \mathrm{kbps}$ (F3) in the lux ${ }^{\mathrm{sec}}$ recombinants confirmed the presence of the intact operon. The absence of any signal of unexpected size indicated that homologous recombination had occurred at the correct target locus in all cases.

In order to phenotypically characterize the lux mutants, they were grown on various BG11-based media (Figure 2D). When spotted on BG11 plates with $5 \mathrm{mM}$ glucose and cultured without selection pressure, all $l u x^{\text {prim }}$ and $l u x^{\text {sec }}$ mutant strains grew normally. The lux $x^{\text {prim }}$ cells resulting from the first round of recombinants grew in the presence of kanamycin. Furthermore, the presence of $5 \%$ sucrose in the medium was lethal for the lux $x^{\text {prim }}$ strains, confirming the functionality of the negative selection marker. Conversely, all the $\operatorname{lu} x^{\sec }$ strains were sensitive to kanamycin, as they had lost the resistance marker, and grew on BG11 supplemented with $5 \%$ sucrose because they no longer harboured the $s a c B$ gene. None of the strains could grow on BG11 supplemented with both kanamycin and sucrose, as expected. This confirms that no integration of the whole vector, also bearing a kanamycin resistance gene on the backbone, via a single crossover event, took place during the first recombination step.

The $\operatorname{lu} x A B$ operon codes for two proteins that are both required for the expression of luminescence, a phenotype not naturally present in Synechocystis. In lux $x^{\text {prim }}$ mutants the full-length $\operatorname{luxB}$ gene is not fused to a functional promoter; therefore, the LuxB subunit of the luciferase cannot be synthesized. The second recombination (in $l u x^{s e c}$ mutants) is expected to reconstitute the entire $\operatorname{lux} A B$ operon under the regulation of $\mathrm{P}_{p s b A 2}$, thus allowing the expression and assembly of the functional enzyme. To confirm this, all the spotted strains were tested for luminescence (Figure 2B, lower panel). Strong luciferase activity was detectable only in the $l u x^{s e c}$ strains carrying the reconstituted $\operatorname{lu} x A B$ operon, and almost absent in all the lux $x^{\text {prim }}$ mutants. A faintly luminescent background was always detectable in the first-round recombinants, possibly due to readthrough transcription of $l u x B$ from the nptI promoter. To better quantify the luciferase activity, light emission was measured for the ten $\operatorname{lu} x^{\text {prim }}$ strains used for second recombination and ten $l u x^{s e c}$ independent recombinants for each of them (Table 2). The luminescence values relative to the untransformed WT Synechocystis strain clearly indicated a gain of function in all the $\operatorname{lu} x^{\sec }$ strains, albeit with a certain inter-strain variability. The level of luciferase activity in the lux $x^{\text {prim }}$ transformants ranged between 1.5- and 2.5-fold higher than in the WT, while in the second-round recombinants it was at least ten times higher than in WT.

\section{Confirmation of the strategy: 2 . The psaA gene}

As photosynthesis is one of the core research areas in which cyanobacteria are extensively used, the same strategy was used to replace the Synechocystis psaA (Syn psaA) gene with the corresponding homolog from the green plant Arabidopsis thaliana (At psaA). The psaA gene codes for PsaA, a core subunit of PSI [25], and in both cyanobacteria and plants it is part of the $p s a A / p s a B$ operon. The two genes are regulated by the $\mathrm{P}_{p s a A}$ promoter located upstream of $p s a A$, and are separated by an intergenic region of 246 base pairs. By transforming the glucose-tolerant WT Synechocystis strain with the vector pDSpsaA, analogously to the Lux experiment described above, knockout ( $\left.p s a A^{\text {prim }}\right)$ and replacement $\left(p s a A^{s e c}\right)$ mutants were subsequently obtained (Figure $3 \mathrm{~A}$ ). The first recombination event yielded colonies in which the integrated construct replaced the endogenous psaA gene, generating the knockout line $p s a A^{\text {prim }}$. The replacement in $p s a A^{\text {sec }}$ affected only the psaA coding sequence and left the surrounding genomic regions unchanged, thus retaining all the endogenous regulatory elements intact. In the $p s a A^{\text {prim }}$ mutant, the integrated $A t$ psaA gene was split into two parts and therefore non-functional. Moreover, also expression of PsaB can be expected to be impaired, because (i) the construct integrated between the common promoter 
Table 2 Luciferase activity relative to $O D_{730}$ in Synechocystis lux ${ }^{\text {prim }}$ and lux ${ }^{\text {sec }}$ mutants

\begin{tabular}{|c|c|c|c|c|c|c|c|c|c|c|}
\hline & $\# 1$ & $\# 2$ & \#3 & $\# 4$ & $\# 5$ & \#6 & \#7 & $\# 8$ & $\# 9$ & $\# 10$ \\
\hline lux & $2.6 \pm 1.4$ & $2.3 \pm 0.4$ & $1.8 \pm 0.4$ & $2 \pm 1.2$ & $2.1 \pm 0.7$ & $1.8 \pm 0.5$ & $2.2 \pm 1.1$ & $1.7 \pm 0.6$ & $1.5 \pm 0.8$ & $1.5 \pm 1$ \\
\hline $\operatorname{lux} x^{\mathrm{sec}}(1-10)$ & $17.3 \pm 3.9$ & $61.8 \pm 11.3$ & $16.6 \pm 3.6$ & $24.1 \pm 6.5$ & $15.3 \pm 5.1$ & $23.2 \pm 9.6$ & $22.7 \pm 9.6$ & $10.4 \pm 3.2$ & $10.6 \pm 2.9$ & $13.1 \pm 3.8$ \\
\hline
\end{tabular}

For each strain, the first recombinant and ten independent second recombinants were analyzed. Each suspension was measured in duplicate and the assay was repeated twice with independently grown cultures. Average luminescence values of the mutants are relative to background signal in the untransformed WT Synechocystis strain, which value was set to 1 .

and the $p s a B$ gene, and (ii) disruption of psaA leads anyhow to the complete lack of any functional PSI, as both PsaA and PsaB cannot accumulate [26]. In consequence, the $p s a A^{\text {prim }}$ strain showed a severe phenotype, since it lacks the PsaA and PsaB proteins, the loss of which prevents the accumulation of any PSI complex [26] and accounts for the turquoise-blue colour of the cells, their high light sensitivity and inability to perform photosynthesis. The absorption spectrum of the mutants showed a complete depletion of chlorophyll $a$ (Chl) with the only presence of the phycobilisome chromophore phycocyanin $(\mathrm{PC})$, responsible for the "blue" phenotype (Figure 3B). After complete segregation of the first replacement, removal of the selective pressure led to the second recombination event and subsequent negative selection on sucrose-containing medium yielded colonies that had lost the nptI-sacB cassette and carried the reconstituted At psaA gene in place of the endogenous one. These $p s a A^{s e c}$ mutants were also bluish, having a drastically reduced $\mathrm{Chl} / \mathrm{PC}$ ratio with respect to the wild-type, but they were able to accumulate more chlorophyll than the psaA prim strain (Figure 3B). The second-round recombinants were not as light sensitive as the first-round recombinants, and they could grow under normal light conditions.

To confirm correct segregation of the introduced sequences in these mutants, genomic PCR was performed using three primer pairs (Figure $3 \mathrm{C}$ ). The region (2 kbps) spanning the upstream homologous region used for integration of the construct (HR1) and the endogenous psaA gene could be amplified with the primer pair P7 + P8 only in WT, confirming that in both mutant strains the knockout was complete. In $p s a A^{\text {prim }}$, but not in WT and in the $p s a A^{s e c}$ mutant, the primer pair P7 + P2 generated an amplicon (3 kbps) spanning HR1, the flanking 5' At $p s a A$ segments and the 5 ' region of the $n p t I-s a c B$ cassette. Primers P9 and P10 amplified the fully reconstituted psaA gene from A. thaliana, generating a PCR product of $2.2 \mathrm{kbps}$ that was detectable only in the psaA $A^{\text {sec }}$ recombinants.

Phenotypic analysis was performed by spotting strains onto different BG11-based media (Figure 3D). The psaA ${ }^{\text {prim }}$ mutant was able to grow on BG11 supplemented with kanamycin, as it harboured the nptI resistance gene but, being PSI-deficient, it only grew heterotrophically in presence of $5 \mathrm{mM}$ glucose and under Light Activated Heterotrophic Growth (LAHG) conditions. In LAHG, bacteria are grown heterotrophically in the dark except for a single short exposure to dim light every $24 \mathrm{~h}$, which was suggested to promote the cell division or the progression through the cell cycle [27]. In contrast, under continuous bright light and in the absence of glucose growth of psaA prim was completely inhibited. The $p s a A^{s e c}$ recombinant was unable to grow on kanamycin but could grow on BG11 containing sucrose, having lost the $n p t I-s a c B$ cassette, whereas the presence of $5 \%$ sucrose was lethal to the knockout strain. None of the mutants, as expected, was viable in presence of both kanamycin and 5\% sucrose. Interestingly, $p s a A^{s e c}$ was not light sensitive and was able to grow photoautotrophically on BG11 agar plates containing no glucose. From these results it can be concluded that the At PsaA protein can partially functionally replace the Synechocystis protein, at least to the extent of restoring light tolerance and the ability to grow photoautotrophically.

\section{Discussion}

Targeted gene and genome manipulation via homologous recombination in bacteria relies on the use of marker genes. In marker-less gene replacement, a negative and a positive selection marker are employed, in a process that normally involves two transformation steps with two DNA suicide vectors. In this work, we developed an alternative strategy for marker-less gene replacement in Synechocystis, based on the use of a single plasmid and a single transformation step. The technique was tested by inserting the $\operatorname{lux} A B$ operon from Vibrio fischeri into the neutral receptor site slr0168. To this end the wild-type strain was transformed with the vector pDSlux and the transformation efficiency with this integration vector was in the range of $10^{-5}$, while the average frequency of the second recombination event was $10^{-6}$ (see Table 1). This ten-fold difference could be due to the fact that the first recombination event involves a double crossover between the genomic DNA and the plasmid vector, whereas the second recombination presumably involves a single intra-genomic crossover [23]. Thus, a difference in efficiency between these two recombination mechanisms could explain the results obtained. Secondary recombinants were easily obtained with 509 bps (psaA) and 1055 bps (luxAB) of homologous regions; however, the exact impact of the length of 


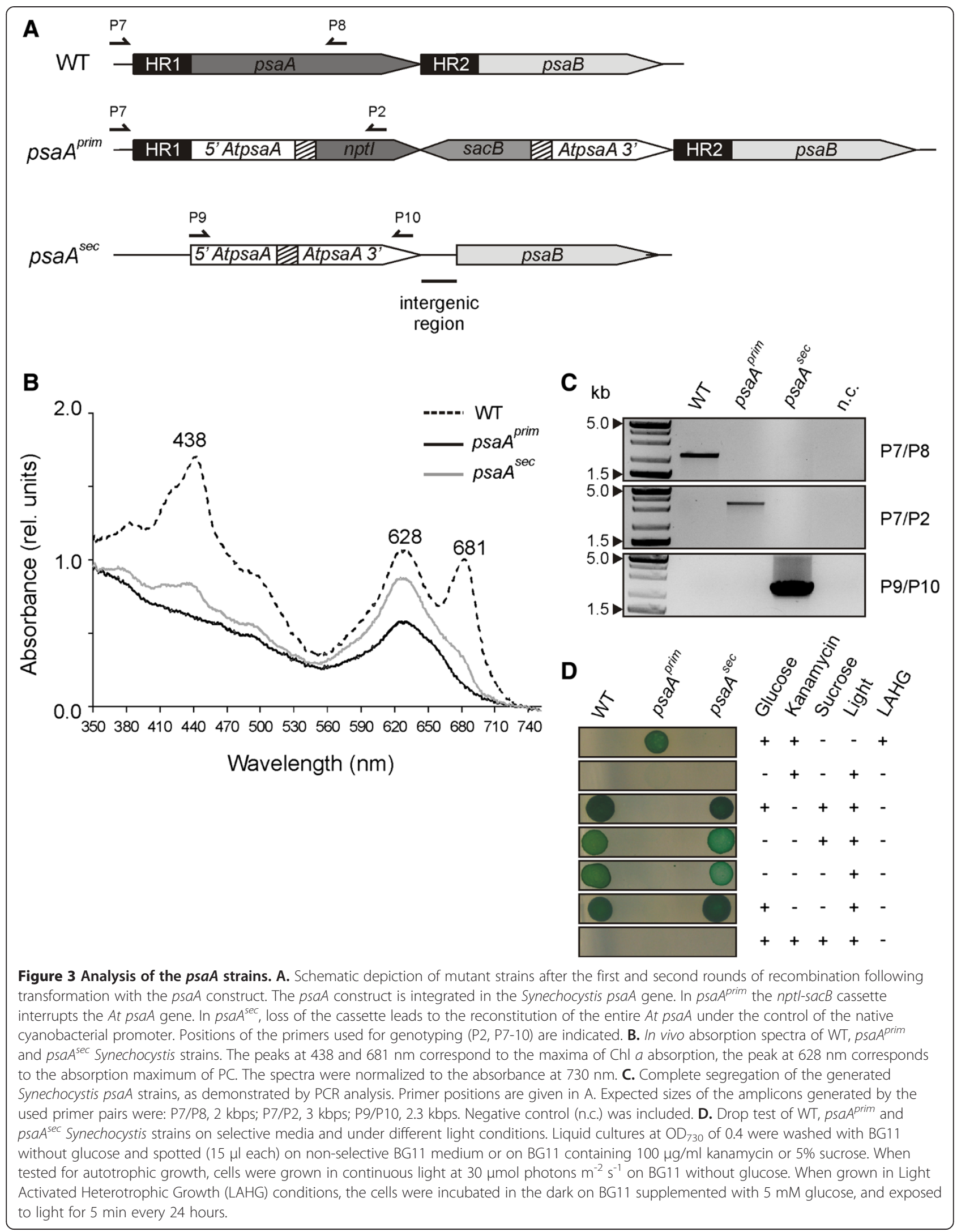


the homologous region on recombination frequency remains to be determined. In addition, other factors like position and relative concentration of the homology regions might contribute to the difference in efficiency [28].

In comparison with standard procedures, the singlestep marker-less gene replacement strategy described here has several advantages. The requirement of only one vector for each gene replacement makes the cloning procedure faster and more facile, especially when one needs to sequentially replace many genes. Our singlestep approach merely requires alternative selective growth conditions in addition to the transformation step, although this flexibility requires that the constructs must be assembled from numerous DNA fragments - six (plus the vector backbone) in the case of pDSlux, for instance. However, the increased complexity of the plasmid vectors required can easily be accommodated by using large-scale modular cloning technologies like the Golden Gate [29], the Gibson [30] or the BioBrick [31] assembly systems. Another issue that can arise during gene manipulation concerns the cloning of genes that are toxic to $E$. coli. Also in this regard, our strategy is advantageous, because the interruption of the GOI sequence by the intervening $n p t I-s a c B$ cassette inactivates it, thus avoiding the problem of toxicity. Only the second recombination in the Synechocystis host cell reconstitutes both the gene sequence and function. Also for studies of biological functions for which two genes are necessary, the single-step gene replacement strategy can constitute a suitable experimental tool, as shown for the lux $A B$ operon. In the lux $x^{\text {prim }}$ mutants, interruption of this operon by the double selection cassette leads to impairment of the luciferase activity, as $\operatorname{lu} x B$ cannot be transcribed and therefore the heterodimeric enzyme cannot be assembled. Only after removal of the cassette by the second recombination event is the integrity of the dicistronic $\operatorname{lux} A B$, and with it the luciferase activity restored, as shown in Figure 2A and Table 2. Some background luminescence was observed in the lux $x^{\text {prim }}$ recombinants, which could be due to read-through transcription of $l u x B$ from the $n p t I$ promoter. Thus, by using only one vector and performing a single transformation step it was possible to show that both $\operatorname{lu} x A$ and $\operatorname{lu} x B$ are necessary for luciferase function, as previously described $[32,33]$. These results confirm that, when our strategy is used to analyse a gain of function, the new function can be expected to arise only in the second recombinants and not in the first mutant strains, which can therefore serve as ideal controls.

The single-step strategy was also employed to replace the psaA gene from Synechocystis with the homolog from Arabidopsis thaliana, which resides in the plastid genome of the plant. The PsaA subunit, together with $\mathrm{PsaB}$, constitutes the dimeric core of PSI, and is highly conserved among photosynthetic organisms from cyanobacteria to flowering plants [34]. PsaA is an essential component of PSI and is necessary for its assembly, accumulation and function, and therefore essential for photoautotrophic growth of both plants and cyanobacteria. Knockout of the psaA gene is lethal to higher plants, while genetic inactivation of the PSI reaction centre in Synechocystis generates mutants that are able to survive heterotrophically under LAHG conditions, although they have a severe phenotype and are unable to survive in the light. In the $p s a A^{s e c}$ mutants, replacement of the endogenous $p s a A$ gene with the $A$. thaliana homolog led to a partial complementation of the psaA $A^{\text {prim }}$ knockout phenotype (Figure 3B,D). Because this is most likely due to a partial functional substitution of the endogenous PsaA protein by its Arabidopsis counterpart, the degree of PSI assembly and function the psa $A^{\text {sec }}$ mutants needs to be analysed in future experiments. Also in this case, using the single-step gene replacement strategy, both the knockout and replacement lines were generated using a single vector and a single transformation. In the case of essential genes like psaA the one-vector strategy confers an additional advantage because mutants with severe phenotypes can be difficult to grow in liquid cultures and therefore their transformation can be challenging. This alternative gene replacement strategy makes it possible to obtain a second recombination event simply by releasing the positive selective pressure on the segregated transformants and applying negative selection in an alternating manner.

\section{Conclusions}

Taken together, the implementation of a double recombination event by a simple stepwise change of growth conditions can be exploited to address various biological issues and could also be employed for large-scale gene replacement approaches. Thanks to the tight counterselection against incomplete second-round recombinants and transformants bearing multiple genomic integrations, the method could indeed be applied in partially automated systems.

\section{Methods}

\section{Bacterial strains and growth conditions}

The bacterial strains used are described in Table 3. E. coli $\mathrm{DH} 5 \alpha$ cultures were grown in lysogeny broth (LB) medium at $37^{\circ} \mathrm{C}$ and shaken at $225 \mathrm{rpm}$. Unless otherwise indicated, wild-type (glucose-tolerant) and mutant Synechocystis sp. PCC 6803 strains were grown at $25^{\circ} \mathrm{C}$ and under continuous illumination at $30 \mu \mathrm{mol} \mathrm{m} \mathrm{m}^{-2} \mathrm{~s}^{-1}$ in BG11 medium containing $5 \mathrm{mM}$ glucose [35]. Liquid cultures were shaken at $120 \mathrm{rpm}$. For growth on plates, $1.5 \%(\mathrm{w} / \mathrm{v})$ agar and $0.3 \%(\mathrm{w} / \mathrm{v})$ sodium thiosulfate were added to the BG11 medium. The psaA $A^{\text {prim }}$ mutant strain 
Table 3 Bacterial strains and plasmids used in this study

\begin{tabular}{|c|c|c|c|}
\hline Strain/plasmid & Characteristics & Selection markers & Source \\
\hline \multicolumn{4}{|l|}{ E. coli } \\
\hline $\mathrm{DH} 5 \mathrm{a}$ & Competent cells & & \\
\hline \multicolumn{4}{|l|}{ Synechocystis } \\
\hline WT & WT Synechocystis sp PCC 6803, glucose tolerant & $\operatorname{kan}^{\mathrm{S}}, \operatorname{suc}^{\mathrm{R}}$ & $\begin{array}{l}\text { H. Pakrasi (Washington } \\
\text { University, St. Louis) }\end{array}$ \\
\hline lux prim & luxAB operon, interrupted by nptl-sacB cassette, replacing slr0168 ORF & $\operatorname{kan}^{R}, \operatorname{suc}^{S}$ & This study \\
\hline $14 x^{s e c}$ & Intact luxAB operon replacing slr0168 ORF & $\operatorname{kan}^{\mathrm{S}}, \operatorname{suc}^{\mathrm{R}}$ & This study \\
\hline psaA $A^{\text {prim }}$ & At psaA gene, interrupted by nptl-sacB cassette, replacing endogenous psaA & kan $^{R}$, suc ${ }^{S}$, heterotroph & This study \\
\hline$p s a A^{s e c}$ & Intact At psaA gene replacing endogenous psaA & kan $^{S}$, suc $^{R}$, photoautotroph & This study \\
\hline \multicolumn{4}{|l|}{ Plasmids } \\
\hline pGEM-T Easy & Backbone for pDSpsaA & $a m p^{R}$ & Promega, Madison, WI \\
\hline pRL250 & $n p t /-s a c B$ double selection cassette, $s a c B$ gene from Bacillus subtilis & $\operatorname{kan}^{\mathrm{R}}, \operatorname{suc}^{\mathrm{S}}$ & $\begin{array}{l}\text { P. Wolk (Michigan } \\
\text { State University) }\end{array}$ \\
\hline plCH69822 & Destination vector for Golden Gate cloning & $\operatorname{kan}^{\mathrm{R}}$ & $\begin{array}{l}\text { E. Weber (Icon Genetics } \\
\text { GmbH, Halle) }\end{array}$ \\
\hline pRL1063a & luxAB operon from Vibrio fischeri & $S m^{R}$ & $\begin{array}{l}\text { P. Wolk (Michigan } \\
\text { State University) }\end{array}$ \\
\hline pDSlux & $\begin{array}{l}\text { plCH69822 with nptl-sacB cassette from pRL250, luxAB operon from } \\
\text { pRL1063a, Syn psbA2 promoter and slr0168 flanking regions }\end{array}$ & $\operatorname{kan}^{\mathrm{R}}, \mathrm{suc}^{\mathrm{S}}$ & This study \\
\hline pDSpsaA & $\begin{array}{l}\text { plCH69822 with nptl-sacB cassette from pRL250, At psaA gene } \\
\text { and Syn psbA2 promoter and slr0168 flanking regions }\end{array}$ & $\operatorname{kan}^{R}, \operatorname{suc}^{S}$ & This study \\
\hline
\end{tabular}

Abbreviations: Syn Synechocystis, At Arabidopsis, R resistant, S sensitive.

was grown under Light-Activated Heterotrophic Growth (LAHG) conditions (i.e. in the presence of glucose and in darkness, interrupted only by a 5 -min exposure light each day) as described [27].

For positive selection of mutants, increasing concentrations of kanamycin (10 to $100 \mu \mathrm{g} / \mathrm{ml}$ ) were added to the medium. For negative selection, BG11 containing 5\% $(w / v)$ sucrose was used.

\section{Generation of plasmids}

All DNA techniques such as plasmid isolation, restriction and ligation were performed according to standard protocols [36]. Synechocystis sequences were obtained from Cyanobase (http://genome.kazusa.or.jp/cyanobase/ Synechocystis). All the plasmids used are listed in Table 3. The fragments used in the construction of the plasmids pDSlux and pDSpsaA were amplified by PCR and purified from 1\% agarose gel. Primers are listed in Additional file 2.

In case of pDSlux, the amplified segments were assembled into the final construct using the one-step Golden Gate Shuffling cloning strategy [29] and the plasmid pICH69822 as destination vector. The Synechocystis slr0168 ORF [37] [GenBankID: 954899] was used as a neutral receptor site for stable integration of the pDSLux vector into the genome. To generate the plasmid, the genomic regions upstream and downstream of slr0168 ( 1 kbps each, HR1 and HR2 respectively) were amplified by PCR and used as flanking regions for homologous recombination. The dicistronic $\operatorname{lu} x A B$ operon from Vibrio fischeri, derived from the pRL1063a vector [38], is composed of the $\operatorname{lu} x A$ and $\operatorname{lux} B$ genes, and encodes the heterodimeric luciferase enzyme which, in presence of its substrate analogue decanal, produces a luminescent product. The operon was placed under the control of the strong Synechocystis psbA2 promoter $\left(\mathrm{P}_{p s b A 2}\right)$ [39] by inserting it upstream of $\operatorname{luxA}$. Two $\operatorname{lux} A B$ amplicons were generated, the first starting at position +1 of $\operatorname{lu} x A$ and ending at position +460 of $\operatorname{lux} B$ and the second starting at position +856 of $\operatorname{lux} A$ and ending at the 3' end of $\operatorname{luxB}$. Thus, the sequence overlap between the two fragments is 1055 base pairs long. In the final construct, the two amplicons are separated by the nptI-sacB double selection cassette, amplified from plasmid pRL250.

The pDSpsaA plasmid was constructed, using overlapping PCR and subsequent standard cloning steps, to replace the Synechocystis psaA gene with the chloroplast-encoded homolog from Arabidopsis thaliana (At psaA). To generate the vector, the $n p t I-s a c B$ cassette was excised from the pRL250 plasmid with BamHI and cloned into the pGEM-T Easy vector (Promega, Madison, Wisconsin) according to the manufacturer's instructions. The regions upstream and downstream ( 500 bps each, HR1 and HR2 respectively) of the Synechocystis psaA gene (slr1834) [GenBank ID: 954060] 
were used as homologous sequences for gene replacement. The At psaA (ArthCp022) [GenBank ID: 844768] coding sequence was placed under the control of the endogenous Synechocystis psaA promoter (contained in HR1), also retaining the native intergenic region of the cyanobacterial $p s a A / p s a B$ operon and the initial part of the native psaB (slr1835, contained in HR2) [GenBank ID: 954061]. Two fragments of At psaA were amplified from cDNA, the first $\left(5^{\prime}\right.$ At psaA) covers the stretch from +1 to +1430 , the second ( $3^{\prime}$ At psaA) extends from +922 to the 3' end. The two amplicons, which overlap for 509 base pairs, were assembled with the upstream and downstream flanking regions via overlapping PCR. The downstream amplicon was cloned at the 3' end of the $n p t I-s a c B$ cassette between the SpeI and SacI restriction sites, then the other amplicon was attached upstream of the cassette between the ApaI and NotI restriction sites.

\section{Transformation of Synechocystis and selection of first- round recombinants}

Synechocystis WT was transformed with the vectors pDSlux and pDSpsaA. For each transformation, $10 \mathrm{ml}$ of growing cells at an $\mathrm{OD}_{730}$ of 0.4 were harvested by centrifugation and resuspended in 1/20 volume of BG11. The cell number per $\mathrm{ml}$ was calculated from $\mathrm{OD}_{730}$ using the formula: $1 \mathrm{OD}_{730}=7 \times 10^{7}$ cells, and $2 \mu \mathrm{g}$ of plasmid DNA per transformation was added to the cells. Transformations were incubated in the light for 5 hours, and shaken the last 3 hours. After addition of fresh BG11 and overnight recovery in the dark, mutants in which the $n p t I-s a c B$ cassette had integrated into the slr0168 open reading frame $\left(l u x^{\text {prim }}\right)$ or replaced the endogenous $p s a A$ gene $\left(p s a A^{\text {prim }}\right)$ were positively selected on BG11 agar plates containing $10 \mu \mathrm{g} / \mathrm{ml}$ kanamycin. Plates bearing cells transformed with pDSlux were incubated in the light, while plated cells transformed with pDSpsaA were incubated under LAHG conditions. The number of transformants obtained was then counted and transformation efficiencies were calculated based on the number of input cells employed. To ensure complete segregation of the mutants, the kanamycin concentration was progressively increased (up to $100 \mu \mathrm{g} / \mathrm{ml}$ ).

\section{Counter-selection and calculation of the frequency of second-round recombinants}

For the second round of recombination and subsequent counter-selection, 10 independent $l u x^{\text {prim }}$ strains were used. One psaA prim mutant was also selected. Each strain was grown to an $\mathrm{OD}_{730}$ of 1 in liquid BG11 containing $100 \mu \mathrm{g} / \mathrm{ml}$ kanamycin. A $500-\mu \mathrm{l}$ sample was taken from each of these cultures, and cells were pelleted, washed and resuspended in 10 volumes of BG11 without antibiotic. The cell number per $\mathrm{ml}$ was calculated as described before. As Synechocystis cells contain multiple copies of the genome, liquid cultures were grown for 5 days without selection in order to allow them to lose all copies of $s a c B$. Then 2-ml samples of each liquid culture were plated on BG11 solid medium containing $5 \%$ sucrose. Plates were incubated in dim light $\left(5 \mu \mathrm{mol}\right.$ photons $\left.\mathrm{m}^{-2} \mathrm{~s}^{-1}\right)$ in the case of the recombinants derived from $p s a A^{\text {prim }}$, in normal light for those obtained from the lux mutants, and the frequency of second-round recombination was determined. Five lux $x^{\text {prim }}$ mutants, and two mutants that had undergone second-round recombination $\left(l u x^{s e c}\right)$ deriving from each of them, were selected and used for subsequent analyses. One second-round recombinant deriving from a psaA $A^{\text {prim }}$ mutant ( $p s a A^{s e c}$ ) was also selected and further grown under normal light conditions.

Genomic PCR was used to confirm the complete segregation of first- and second-round recombinants. Genomic DNA was extracted from Synechocystis using the xanthogenate method [40]. Primers used to genotype the lux mutants were: Lux1 FW (P1), DS_nptI RV (P2), sac_S2 (P3) and Lux2 RV (P4), slr0168 DR FW (P5), slr0168 DW RV (P6). The primers used for genotyping of the $p s a A$ mutants were: $p s a A$ gUR FW (P7), DS_nptI RV (P2), psaA_Syn RV (P8), A2 FW (P9) and A3 RV (P10).

\section{Southern analysis}

For Southern analysis, $5 \mu \mathrm{g}$ of genomic DNA were digested with XmaI endonuclease. The fragments were electrophoretically separated on a $0.8 \%$ agarose gel in $0.5 \times$ TBE $(40 \mathrm{mM}$ Tris-HCl, $\mathrm{pH} 8.3,45 \mathrm{mM}$ boric acid, $1 \mathrm{mM}$ EDTA) and then blotted onto nylon membrane (Hybond N+; GE Healthcare, http://www.gehealthcare. com/). The DS_lux probe (1.7 kbps) was amplified with primers Lux1 FW and Lux1 RV. The probe was labeled using the random-priming method with ${ }^{32} \mathrm{P}[\mathrm{dCTP}]$. Hybridization was carried out overnight at $67^{\circ} \mathrm{C}$. Signals were detected with a phosphoimager (Typhoon; GE Healthcare, http://www.gehealthcare.com/).

\section{Luciferase assay}

Luciferase activity was induced by the addition of decanal (an analogue of the luciferase substrate luciferin) to the cyanobacterial suspension to a final concentration of $1 \mathrm{mM}$ (from $50 \mathrm{mM}$ decanal in methanol/water $50 \%$, $\mathrm{v} / \mathrm{v}$ stock). The reaction was incubated for $15 \mathrm{~min}$ with mild shaking and luminescence was measured with a microplate reader (Safire ${ }^{2}$; Tecan, http://www.tecan. $\mathrm{com} /$ ) at room temperature. Luminescence values are expressed relative to the optical density of the cell suspension at $730 \mathrm{~nm}$, also measured with the microplate reader. Each suspension was measured in duplicate and the assay was repeated twice with independently grown cultures. 
To assay luciferase activity on solid medium, the decanal solution was added to cells grown on agar plates, and luminescence was detected with the FUSION FXT imaging system (Peqlab, http://www.peqlab.com/).

\section{Bacterial whole-cell absorbance spectra}

Absorbance spectra of whole Synechocystis cells were recorded using a spectrophotometer. Cells were harvested, washed and resuspended in BG11 liquid medium to a final $\mathrm{OD}_{730}$ of 0.5 . Their absorbance spectra were recorded between 350 and $750 \mathrm{~nm}$ and corrected for the light scattering at $730 \mathrm{~nm}$.

\section{Additional files}

\section{Additional file 1: Schematic depiction of the classical double recombination strategy.}

Additional file 2: List of primers used in this study. Restriction sites are indicated by bold characters. The Bsal-generated sticky ends used for Golden Gate Shuffling assembly of the pDSlux constructs are underlined. In the case of the pDSpsaA plasmid, regions used for assembly with overlapping PCR are indicated in italics. Abbreviations: Syn, Synechocystis; At, Arabidopsis.

\section{Competing interests}

The authors declare that they have no competing financial interests.

\section{Authors' contributions}

SV carried out the experimental work and drafted together with TR and DL the manuscript. All authors participated in the design of the study. DL conceived the study. All authors read and approved the final manuscript.

\section{Acknowledgements}

We thank Prof. Dr. Peter Wolk (MSU-DOE Plant Research Laboratory Michigan State University, U.S.A.) for providing the vector pRL250, Dr. Sylvestre Marillonet (Icon Genetics $\mathrm{GmbH}$, Halle, Germany) for providing the Golden Gate vector plCH69822 and Prof. Dr. Annegret Wilde (Molecular Genetics, Albert-Ludwigs-Universität Freiburg, Germany) for providing the luxAB operon from the vector pRL1063a. We thank Paul Hardy for critical reading of the manuscript.

Received: 4 November 2013 Accepted: 3 January 2014

Published: 8 January 2014

\section{References}

1. Whatley JM, Whatley FR: Chloroplast evolution. New Phytol 1981, 87:233.

2. Golden SS, Ishiura M, Johnson CH, Kondo T: Cyanobacterial circadian rhythms. Annu Rev Plant Physiol Plant Mol Biol 1997, 48:327-354

3. Angermayr SA, Hellingwerf K, Lindblad P, de Mattos MJ: Energy biotechnology with cyanobacteria. Curr Opin Biotechnol 2009, 20:257-263.

4. Hess WR: Cyanobacterial genomics for ecology and biotechnology. Curr Opin Microbiol 2011, 14:608-614.

5. Kaneko T, Sato S, Kotani H, Tanaka A, Asamizu E, Nakamura Y, Miyajima N, Hirosawa M, Sugiura M, Sasamoto $S$, et al: Sequence analysis of the genome of the unicellular cyanobacterium Synechocystis sp. strain PCC 6803. II. Sequence determination of the entire genome and assignment of potential protein-coding regions (supplement). DNA Res 1996, 3:185-209.

6. Williams JGK: Construction of specific mutations in photosystem II photosynthetic reaction center by genetic-engineering methods in Synechocystis 6803. Methods Enzymol 1988, 167:766-778.

7. Dühring U, Ossenbühl F, Wilde A: Late assembly steps and dynamics of the cyanobacterial photosystem I. J Biol Chem 2007, 282:10915-10921.

8. Xu W, Wang YC, Taylor E, Laujac A, Gao LY, Savikhin S, Chitnis PR: Mutational analysis of photosystem I of Synechocystis sp PCC 6803: the role of four conserved aromatic residues in the j-helix of PsaB. PLoS One 2011, 6:e24625.

9. Vermaas WFJ, Williams JGK, Rutherford AW, Mathis P, Arntzen CJ: Genetically engineered mutant of the cyanobacterium Synechocystis 6803 lacks the photosystem II chlorophyll-binding protein CP-47. Proc Natl Acad Sci USA 1986, 83:9474-9477.

10. Vermaas WFJ, Williams JGK, Arntzen CJ: Sequencing and modification of psbB, the gene encoding the CP-47 protein of photosystem II, in the cyanobacterium Synechocystis 6803. Plant Mol Biol 1987, 8:317-326.

11. Nixon PJ, Rögner $M$, Diner BA: Expression of a higher plant $p s b A$ gene in Synechocystis 6803 yields a functional hybrid photosystem II reaction center complex. Plant Cell 1991, 3:383-395.

12. He QF, Schlich T, Paulsen H, Vermaas W: Expression of a higher plant lightharvesting chlorophyll a/b-binding protein in Synechocystis sp PCC 6803. Eur J Biochem 1999, 263:561-570.

13. Labarre J, Chauvat F, Thuriaux P: Insertional mutagenesis by random cloning of antibiotic-resistance genes into the genome of the cyanobacterium Synechocystis strain PCC 6803. J Bacteriol 1989, 171:3449-3457.

14. Ried JL, Collmer A: An npt/-sacB-sacR cartridge for constructing directed, unmarked mutations in Gram-negative bacteria by marker exchangeeviction mutagenesis. Gene 1987, 57:239-246.

15. Fouet A, Arnaud M, Klier A, Rapoport G: Characterization of the precursor form of the exocellular levansucrase from Bacillus subtilis. Biochem Biophys Res Commun 1984, 119:795-800

16. Steinmetz M, Lecoq D, Aymerich S, Gonzytreboul G, Gay P: The DNA sequence of the gene for the secreted Bacillus subtilis enzyme levansucrase and its genetic-control sites. Mol Gen Genet 1985, 200:220-228.

17. Gay P, Lecoq D, Steinmetz M, Ferrari E, Hoch JA: Cloning structural gene $s a c B$, which codes for exoenzyme levansucrase of Bacillus subtilis: expression of the gene in Escherichia coli. J Bacteriol 1983, 153:1424-1431.

18. Cai YP, Wolk CP: Use of a conditionally lethal gene in Anabaena sp strain PCC 7120 to select for double recombinants and to entrap insertion sequences. J Bacteriol 1990, 172:3138-3145.

19. Matsuoka M, Takahama K, Ogawa T: Gene replacement in cyanobacteria mediated by a dominant streptomycin-sensitive rps 12 gene that allows selection of mutants free from drug resistance markers. Microbiology 2001, 147:2077-2087.

20. Funatsu G, Wittmann HG: Ribosomal proteins. XXXIII. Location of aminoacid replacements in protein $\mathrm{S} 12$ isolated from Escherichia coli mutants resistant to streptomycin. J Mol Biol 1972, 68:547.

21. Timms AR, Steingrimsdottir $H$, Lehmann AR, Bridges BA: Mutant sequences in the $r p s L$ gene of Escherichia coli B/r: mechanistic implications for spontaneous and ultraviolet light mutagenesis. Mol Gen Genet 1992, 232:89-96.

22. Lalioti MD, Heath JK: A new method for generating point mutations in bacterial artificial chromosomes by homologous recombination in Escherichia coli. Nucleic Acids Res 2001, 29:e14.

23. Clerico EM, Ditty JL, Golden SS: Specialized techniques for site-directed mutagenesis in cyanobacteria. Methods Mol Biol 2007, 362:155-171.

24. Zang XN, Liu B, Liu SM, Arunakumara KKIU, Zhang XC: Optimum conditions for transformation of Synechocystis sp PCC 6803. J Microbiol 2007, 45:241-245

25. Chitnis PR: PHOTOSYSTEM I: function and physiology. Annu Rev Plant Physiol Plant Mol Biol 2001, 52:593-626.

26. Smart $L B$, Anderson $S L$, Mclntosh $L$ : Targeted genetic inactivation of the photosystem I reaction centre in the cyanobacterium Synechocystis sp. PCC 6803. EMBO J 1991, 10:3289-3296.

27. Anderson SL, Mclntosh L: Light-activated heterotrophic growth of the cyanobacterium Synechocystis sp. strain PCC 6803: a blue-light-requiring process. J Bacteriol 1991, 173:2761-2767.

28. Kufryk Gl, Sachet M, Schmetterer G, Vermaas WFJ: Transformation of the cyanobacterium Synechocystis sp PCC 6803 as a tool for genetic mapping: optimization of efficiency. FEMS Microbiol Lett 2002, 206:215-219.

29. Engler C, Gruetzner R, Kandzia R, Marillonnet S: Golden gate shuffling: a one-pot DNA shuffling method based on type lls restriction enzymes. PLoS One 2009, 4:e5553.

30. Gibson DG, Young L, Chuang RY, Venter JC, Hutchison CA, Smith HO: Enzymatic assembly of DNA molecules up to several hundred kilobases. Nat Methods 2009, 6:343-U341.

31. Shetty RP, Endy D, Knight TF Jr: Engineering BioBrick vectors from BioBrick parts. J Biol Eng 2008, 2:5. 
32. Engebrecht J, Nealson K, Silverman M: Bacterial bioluminescence: isolation and genetic analysis of functions from Vibrio fischeri. Cell 1983, 32:773-781

33. Foran DR, Brown WM: Nucleotide sequence of the LuxA and LuxB genes of the bioluminescent marine bacterium Vibrio fischeri. Nucleic Acids Res 1988, 16:777.

34. Amunts A, Nelson N: Functional organization of a plant Photosystem I: evolution of a highly efficient photochemical machine. Plant Physiol Biochem 2008, 46:228-237.

35. Rippka R, Deruelles J, Waterbury JB, Herdman M, Stanier RY: Generic assignments, strain histories and properties of pure cultures of cyanobacteria. J Gen Microbiol 1979, 111:1-61.

36. Sambrook J, Russell DW: Molecular cloning: a laboratory manual. New York: Cold Spring Harbor Press; 2001

37. Kunert A, Hagemann M, Erdmann N: Construction of promoter probe vectors for Synechocystis sp. PCC 6803 using the light-emitting reporter systems Gfp and LuxAB. J Microbiol Methods 2000, 41:185-194.

38. Wolk CP, Cai YP, Panoff JM: Use of a transposon with luciferase as a reporter to identify environmentally responsive genes in a cyanobacterium. Proc Natl Acad Sci USA 1991, 88:5355-5359.

39. Eriksson J, Salih GF, Ghebramedhin H, Jansson C: Deletion mutagenesis of the 5' psbA2 region in Synechocystis 6803: identification of a putative cis element involved in photoregulation. Mol Cell Biol Res Commun 2000, 3:292-298.

40. Tillett $D$, Neilan BA: Xanthogenate nucleic acid isolation from cultured and environmental cyanobacteria. J Phycol 2000, 36:251-258.

doi:10.1186/1475-2859-13-4

Cite this article as: Viola et al:: A single vector-based strategy for marker-less gene replacement in Synechocystis sp. PCC 6803. Microbial Cell Factories 2014 13:4.

\section{Submit your next manuscript to BioMed Central and take full advantage of:}

- Convenient online submission

- Thorough peer review

- No space constraints or color figure charges

- Immediate publication on acceptance

- Inclusion in PubMed, CAS, Scopus and Google Scholar

- Research which is freely available for redistribution 